\title{
Jahres-Inhalt 1991, Vol. 14, No. 6, 1991
}

Complete Contents 1991

Editoral

ONKOLOGIE - Warum eine internationale Zeitschrift?

Queißer,W 5

Operative Tumortherapie - weiter nichts?*

Schlag,P 99

Übersichtsarbeiten

Vinorelbin (Navelbine $\left.{ }^{\circledR}\right)$, ein neues semisynthetisches Vincaalkaloid*

Krikorian, A., Breillout, F 7

Ergebnisse der Chirurgie im Rahmen

der multimodalen Therapie des Ösophaguskarzinoms*

Schlag,P 13

Lebertransplantation bei Leberkrebs*

Steffen, R., Neuhaus, P., Blumhardt, G., Bechstein, W.0 100

Chirurgische und adjuvante Maßnahmen zur Reduktion von Lebermetastasen beim Dickdarm-

karzinom*

Schlag,P., $\quad 108$

Die Notwendigkeit weiterer adjuvanter Therapie-studien bei gastrointestinalen Tumoren*

Douglass Jr., H.0 114

Intraperitoneale Chemotherapie und Immuntherapie. Gegenwärtiger Stand und Perspektiven*

Eggermont, A.M.M., Sugarbaker, P.H 123

Systemische Chemotherapie maligner Erkrankungen der Haut

Reinhold, U 137

Fortschritte beim kurativen Einsatz der Chemotherapie bei Kopf-Halskarzinomen*

Volling, P., Schroder, M 205

1st Verapamil eine geeignete Substanz zur Überwindung der Zytostatikaresistenz?*

Harder, S 212

Hormonelle Behandlung des fortgeschrittenen Mammakarzinoms: Therapiesequenzen,

Substanzkombinationen, neue hormonell wirksame Medikamente

Worst, P 218

Proliferationskinetik und Prognose eines Nierenzellkarzinoms*

de Riese, W, Allhoff, E., Werner, M., Stief, C.G.,

Atzpodien, J., Kirchner, H 297

Zur Bewertung von Therapiestudien beim Mammakarzinom

Sauerbrei, W., Schmoor, C, Schumacher, M 303

Immunzytologische Điagnostik des Blasenkarzinoms*

Huland, E., Huland, H385

Therapie beim Larynxkarzinom: Überleben, Tumorkontrolle und Funktion unter besonderer Berücksichtigung der Radiotherapie* 
Glanzmann, C 465

Die bulbuserhaltende Strahlenbehandlung primärer intraokularer Melanome

Müller, R.-P 479

Stellenwert der Knochenmarktransplantation in der Therapie des multiplen Myeloms*

Heyll, A., Aul, C, Runde, V., Thomas, M., Schneider, W 485

Aspekte der Datenerhebung bei Protokollen zur adjuvanten Therapie des kolorektalen

Karzinoms*

Hermanek, P 490

Originalarbeiten

Goserelin als Depot GnRH-Analogon zur Therapie prämenopausaler Patientinnen mit

metastasiertem Mammakarzinom - 5-Jahres-Erfahrung mit Hormon-Folgetherapien*

Kaufmann, M., Jonat, W., Schachner-Wünschmann, E.,

Bastert, G, Maass, H 22

Orale Idarubicintherapie als zytostatische Erstbehandlung bei Patientinnen mit metastasierten

Mammakarzinomen und günstigen Prognosekriterien*

Possinger, K., Wagner, H., Worst, P., Queißer, W., Bremer, K.,

Rieche, K., Klee, M., Westerhausen, M., Donhuijsen-Ant, R.,

Fritze, D., Edler, L., Stiegelbauer, G, Burk, K

31

Phase-II-Studie mit oralem Vinorelbine zur Behandlung des metastasierten Mammakarzinoms*

Queißer, W., Doss, A., Wander, H.E., Bremer, K., Becher, R.,

Rieche, K., Delgado, F.M., Edler, L 35

Zur Therapie des neuropathischen Tumorschmerzes mit Antidepressiva und Antikonvulsiva*

Kloke, M., Höffken, K., Olbrich, H., Schmidt, C.G 40

Funktionelle Kriterien für die Stadieneinteilung und Behandlung der Haarzelleukämie*

Porzsolt, F, Demeter, J., Heimpel, H 44

Wie kann das krankheitsfreie Überleben

von erwachsenen ALL-Patienten mit oder ohne

Transplantation miteinander verglichen werden?*

Messerer, D., Neiß, A., Aydemir, Ü., Hoelzer, D., Arnold, R.. . .53

Operative Therapie bei tumorösem Befall der Wirbelsäule

Venbrocks, R., Donk, R., Hovel, M., Fuhrmann, R 56

544

Jahres-Inhalt 1991

$/ \mathrm{u}$ in Stellenwert von Nachsorgekuren

in der Krankheitsbewältigung von Tumorpatienten

Schmelzle, M., Schwarz, R., Fellhauer, S., Schlag, P

Immunocheniochirurgie (postoperative Immuno-chemotherapie) als angemessene Behandlung des Magenkarzinoms im Stadium III*

Kim, J.-P 148

Intensive intraoperative lokale Chemotherapie bei Lymphknotenmetastasen und

Peritonealkarzinose beim Magenkarzinom*

Takahashi, T., Hagiwara, A., Sawai, K., Kondo, S.,

Yamaguchi, T 152

Insulin und Diazoxid zur Tumortherapie. Ergebnisse einer klinischen Pilot-Studie*

Fink, M., Abenhardt, W., Ostermayr, B., Berger, M 158 
Einfluß von Thymopentin und Isoprinosin auf Parameter der zellulären Immunität bei Patientinnen mit Ovarialkarzinom

Heine, O., Vahrson, H., Santoso, S., Leib, P., Pabst,W 165

Ergebnisse und therapeutische Konsequenzen der Staging Laparotomie beim Morbus Hodgkin

Fuller, E., Gerhartz, H.H., Löffler, M., Pfreundschuh, M.,

Smith, K., König, J., Rühl, H., Rühl, U., Wilmanns, W.,

Diehl,V 171

Rationale und Stellenwert von Präoperativen Therapiemaßnahmen bei Patienten mit lokal

fortgeschrittenem Mammakarzinom*

Jakesz, R., Steger, G., Rainer, H 228

Prognosefaktoren von Patienten mit zerebralen Metastasen in der frühen postoperativen Phase*

Sachsenheimer, W., Bimmler, T., Piotrowski, W 234

Supportive Betreuung terminal krebskranker Patienten zu Hause und in einem Tages-Hospiz

Kleeberg, U.R., Kerekjarto, M., Kaden, FL,

Wagner-Bastemeyer, R., Kur, A., Lehmann, G., Schulz, K.,

Bogan, G., Reichel, L., Erdmann, H 240

Antibiotische Behandlung der Sepsis mit Teicoplanin bei knochenmarktransplantierten

Patienten*

Fauser, A.A., Lang, E., Schmid, J., Dölken, G., Kreisel, W.,

Löhr, G.W 248

Unterschiedliche CKII-Aktivitäten in menschlichen, kolorektalen Schleimhäuten, Adenomen und Karzinomen*

Pistorius, K., Seitz, G., Remberg, K., Issinger, O. G 256

Steigerung der Zytotoxizitat spezifischer monoklonaler Antikörper gegen humane

Karzinomzellen durch GM-CSF in vitro

Staib, L., Link, K.H., Büchler, M 261

Etoposid, Leukovorin und 5-Flourouracil (ELF) beim fortgeschrittenen Magenkarzinom:

Abschließende Ergebnisse einer Phase-II-Studie bei älteren Patienten oder Patienten mit

kardialem Risiko*

Stahl, M., Wilke, H., Preusser, P., Fink, U., Achterrath, W.,

Schöber, C, Köhne-Wömpner, C.H., Harstrick, A.,

Meyer, H. J., Meyer, J., Lenaz, L., Schmoll, H. J 314

Einfluß von Rekombinantem Granulozyten-Makro-phagen Kolonien-stimulierendem Faktor

(GM-CSF) auf Leukopenie bei AIDS: Eine Untersuchung bei sieben Patienten*

Ottmann, O.G., Goebel, F.-D., Ganser. A., Bogner, J.R.,

Seipelt, G., Chatterjee, M., Helm, E.B., Hoelzer, D 319

Chirugische Therapie bei großen Knochenmetastasen im Becken

Spring. W., Dittmer, H 322

Prospektive multizentrische Phase-III-Studie mit Doxifluridin (5'dFUR) versus 5-Flourouracil bei Patienten mit fortgeschrittenem kolorektalen Karzinom*

Schuster, D., Heim, M.E., Dombernowski, P., Wood, C,

Queißer,W 333

Phase-I-Studie mit Miltefosine (Hexadexyl-Phospho-choline; wöchentlich oral) bei

Tumorpatienten* 
Danhauser-Riedl, S., Drozd, A., Zafferani, M., Bruntsch, U.,

Peukert, M., Sindermann, H., Präuer, H.W., Siewert, J.R., Rastetter,

J., Berdel, W.E 392

Phase-II-Studie mit Droloxifen zur Behandlung des metastasierten kolorektalen Karzinoms*

Nienhaus, P., Queißer, W., Heim, M.E., Pechan, R 401

Lokoreginäre versus systemische Chemotherapie beim metastasierten kolorektalen Karzinom -

Effizienz einer additiven Immuntherapie mit Rubratin. Eine prospektiv randomisierte

Pilotstudie*

Hölting, T., Frohmüller, S., Aulenbacher, P., Peukert, M.,

Schlag, P 406

Behandlung des metastasierenden Melanoms mit einem pyrogenen Bakterienlysat. Eine

Pilotstudie*

Kölmel, KE., Vehmeyer, K., Göhring, E., Kuhn, B.,

Wieding,J.U 411

Graduierte WHO-analoge Skalen und Zufriedenheits-Skalen zur Erfassung der Lebensqualität in

klinischen Studien*

Heidemann, E., Kaesberger, J., Herschbach, P.,

Sellschopp, A 419

Unterschiedliche Kinetik des Ansprechens auf Therapie bei Hoch-Risiko- und Niedrig-Risiko-

Patientinnen mit metastasiertem Mammakarzinom*

Porzsolt, F., Meuret, G. (für die regionale Mammakarzinom-

Studiengruppe, Tumorzentrum Ulm) 428

Zytotoxizitat von 16alpha-(125I)Jod-17beta-Östradiol für Ostrogenrezeptor-positive

Mammakarzinomzellen in vitro

Scharl, A., Beckmann, M.W., Schreiber, J.R., Holt, J. A 433

Ergebnisse der Strahlentherapie von Hirntumoren, Radiologische Klinik, Kiel 1970-1979*

May, C, Dühmke, E 498

Undifferenzierte Gliome: Ergebnisse unter Strahlentherapie und Razoxan

Eiter, H, Rhomberg, W 507

Perioperative Chemotherapie des fortgeschrittenen Ösophaguskarzinoms

Blum, M., Nottberg, H, Preusser, P., Bünte, H 514

Jahres-Inhalt 1991

545

Nicht tastbares Mammakarzinom - Histologische Ergebnisse nach präoperativer Markierung von 397 nicht palpablen Läsionen der Mamma

Smola, M.G., Ratschek, M., Hauser, H., Amann, W., Veith, G.,

Ruppitsch, U., Steindorfer, P 518

Psychosomatische Gespräche mit Krebskranken -bewältigungsorientierte Kurzzeittherapie

Leiberich, P., Kreuzer, E., Olbrich, E., Kalden, J. R 524

Laufende klinische Studien

Multizentrische prospektive kontrollierte Studie zur Therapie der chronisch myeloischen

Leukämie. Vergleich von Busulfan, Hydroxyurea und Interferon alpha (Stand April 1990)

Hehlmann, R., Heimpel, H., Heinze, B., Georgii, A., Kolb, H.J.,

Hossfeld, D.K., v. Wussow, P., Hochaus, A., Grießhammer, M.,

Diehl, V., Wickramanayake, P.D., Gallmeier, W.M., Falge, C, 
Essers, U., Bergmann, L., Meyer, P., Walther, F., Kanz, L., Queißer, U., Hohnloser, J., Westerhausen, M., Kleeberg, U.R., Heilein, A., Käbisch, A., Heiss, F., Zimmermann, R., Meuret, G., Tichelli, A., Berdel, W.E., Schmitz, N., Tigges, F.-J., Eimermacher, H., Schmid, L., Löffler, H., Pralle, H., Queißer, W., Burkhardt, R., Ansari, H., Hasford, J 66

5-Fluorouracil in Kombination mit Interferon-alpha 2c bei fortgeschrittenen Magen-DarmKarzinomen Hausmanninger, H., Oentrich, B., Nachbaur, K., Niederwieser, D., Haidinger, R, Huber, C 178

Randomisierte multizentrische Studie zur Behandlung hochmaligner Non-Hodgkin-Lymphome: CHOEP vs. hCHOP/ГVEP*

Köppler, H., Pflüger, K.H., Eschenbach, I., Pfab, R., Birkmann, J., Zeller, W., Steinhauer, E. U., Gropp, C, Oehl, S., Lötzke, E., Kuhn, H., Drings, P., Großmann, H.H.,

Lennert, K., Stein, H., Khoury, M., Heinzelmann, W., Havemann, K 181

Hochmaligne periphere-T-Zell-Lymphome: Therapie-Ergebnisse einer retrospektiven offenen multizentrischen Studie

Kuse, R., Lennert, K., Calavrezos, A., Tiemann, M., Engelhard, M

268

Ansprechraten und Toxizität von Miomycin-C, Mitoxantron und Methotrexat (3M) bei metastiertem Mammakarzinom*

Porzsolt, F., Meuret, G 338

Schmerztherapie: Vergleich zweier Bestrahlungs-schemata bei schmerzhaften

Knochenmetastasen*

Karstens, J.H., Blach, M., Ammon, J 341

Immunzytologische Differenzierung zwischen Leberzellkarzinom und metastasierendem extragonadalem Keimzelltumor

Hastka, J., Bohrer, M.H., Hartung, G., Verbeke, C.S344

Kasuistiken

Erfolgreich durch Radiotherapie behandelte kutane Manifestationen des Morbus Waldenström Latz, D., Mende, U 275

Nachweis von Mycobaxterium tuberculosis bei atypischer Röntgenmorphologie eines kleinzelligen Bronchialkarzinoms unter Zytostatika-Therapie

Heckmayr, M., Gatzemeier, U 346

Ähnliche histologische Muster eines bilateralen malignen Teratoms der Ovarien und eines

früheren Retinoblastoms bei einem Mädchen*

Schroder, W 437

Informationen für die Klinik

Kostenvergleich zwischen stationärer und ambulanter Dauerinfusionstherapie

Löffler, T.M., Weber, F.W 352

Die Klinik für Tumorbiologie an der Universität Freiburg -Ein neues Modell der Krebsforschung Nagel,G.A 441

* diese Arbeit erscheint in englischer Sprache 
Erste Ergebnisse einer Induktionstherapie mit

fraktioniertem Aclacinomycin, Etoposid und

Cytosinarabinosid bei (myelo-)monozytärer Leukämie

in höherem Alter und/oder nach myelodysplastischem

Syndrom

Krieger, O., Kasparu, H., Weber, E., Lutz, D 272

Kurzmîtteilungen

Eine neue Methode zur mehrfachen Injektion von Zytostatika bei Ratten*

Klesper, B., Zywietz, F

74

Zur Frage der Dosierung von Zytostatika in der Onkologie*

Torsten,U., Hiller, H.H., Opri, F., Weitzel,H.K 185

Supplement 1

Übersicht über derzeitige Konzepte in der Psychoneuroimmunologie*

Kappauf,H.W 10

Interaktionen zwischen Immunsystem und zentralem

Nervensystem (I)*

Fischer, J 14

Interaktionen zwischen Immunfunktionen und zentralem Nervensystem (II)*

Yirmiya, R 16

Streßeffekte auf das Immunsystem*

Schulz, K.H 19

Auswirkungen von Verlust und Depression auf Immunmechanismen und Onkogenese*

Beutel, M 30

546

Jahres-Inhalt 1991

Spontanremissionen und unerwartet gunstiger Verlauf*

Kappauf,H.W 32

Therapeutische Schlußfolgerungen aus den psychoneuroimmunologischen

Forschungsergebnissen*

Speidel, $\mathrm{H} \quad 36$

Interventionsmethoden*

Köhle, K 39

Empfehlungen für die Umsetzung der Psychoneuroimmunologie-Forschungsergebnisse in die onkologische Praxis*

Kappauf, H.W., Büntig, W.E 42

* Die Beiträge sind Zusammenfassungen der neun gleichnamigen Konferenzsitzungen. Die Autoren beziehen sich auf die jeweiligen Expertenvorträge und Diskussionsbeiträge. 